Bull. Austral. Math. Soc.

13B30, 13D 30

VoL. 38 (1988) [373-375]

\title{
CONTRACTED PRIMES OF THE COMPLETE RING OF QUOTIENTS
}

\author{
Frederick W. Call
}

The generic closure of the set of primes contracted from the complete ring of quotients of a reduced commutative ring is shown to be just the set of those primes not containing a finitely generated dense ideal. It is also the smallest generically closed, quasi-compact set containing the minimal primes.

In the study of the complete ring of quotients $Q(R)$ of a reduced commutative ring $R$, knowledge of the set

$$
G=\{m \cap R \mid m \in \operatorname{Spec} Q(R)\}
$$

of contracted primes is useful. For example [3, Theorem 4.3], $Q(R)$ is flat if and only if $G=\min R$, the set of minimal primes of $R$. In this note we characterise the generic closure of $G$. Here, $Q(R)$ can be defined as

$$
\lim _{\rightarrow} \operatorname{Hom}(I, R)
$$

with direct limit taken over all dense ideals $I$ of $R$, or as

$$
Q(R)=\{x \in E(R) \mid I x \subseteq R \text { for some dense ideal } I \subseteq R\}
$$

$E(R)$ is the injective envelope of $R, I$ dense means $I$ has zero annihilator in $R$ (see [7] for general considerations). If $H \subseteq \operatorname{Spec} R$, its generic closure is

$$
\bigwedge(H)=\{p \in \operatorname{Spec} R \mid p \subseteq q \in H\}
$$

and $H$ is generically closed if $H=\wedge(H)$. We use only the Zariski topology on the prime spectrum Spec $R$. Let $G_{f}$ be the set of primes of $R$ not containing a finitely generated dense ideal.

Received 15 January, 1987

Copyright Clearance Centre, Inc. Serial-fee code: 0004-9729/88 \$A2.00+0.00. 
TheOREM. For a reduced ring $R, \bigwedge(G)=G_{f}$ and is the smallest generically closed, quasi-compact set of primes containing $\min R$.

Proof: First we note that, in a reduced ring, a finitely generated ideal is dense if and only if it is not contained in any minimal prime (since a minimal prime can contain a finitely generated ideal or its annihilator, but not both). Secondly, $Q(R)$ is a von Neumann regular ring since $R$ is reduced [4, Proposition 2.4.1].

Clearly, $G_{f}$ is generically closed. It is also quasi-compact since the set of primes not containing a finitely generated ideal is a "patch", hence so too is an arbitary intersection since patches form the closed sets of a topology ([2] or [1, Exercise 3.27, 3.28]). To show that $G_{f}$ contains the set $G$ of contracted primes, we suppose $p \notin G_{f}$. Choose a finitely generated dense ideal $I$ containing $p$. Then $I Q(R)$ is dense in $Q(R)$ since $Q(R)$ is an essential extension of $R$. As the only finitely generated (hence generated by an idempotent) dense ideal in a regular ring is the ring itself, it follows that $p$ is not a contracted prime, $G \subseteq G_{f}$, hence $\Lambda(G) \subseteq G_{f}$.

For the reverse inclusion, we begin by proving $\Lambda(G)$ contains all the minimal primes. To this end, suppose $p \in \min R$, but that $p Q(R)=Q(R)$. Write $1=\sum r_{i} x_{i}$, for some $r_{i} \in p$ and $x_{i} \in Q(R)$. Now there exist dense ideals $I_{i}$ of $R$ such that $I_{i} x_{i} \subseteq R$ for each $i$. Let $I$ be the product of the $I_{i}$, also a dense ideal. We have

$$
I=\sum r_{i} I x_{i} \subseteq \sum r_{i} R .
$$

This last ideal is a finitely generated dense ideal contained in the minimal prime $p$, a contradiction to the remark at the begining of the proof. Thus $p Q(R) \neq Q(R)$, that is, $p \in \Lambda(G)$ and $\Lambda(G) \supseteq \min R$. Now let $C$ be any subset of Spec $R$ that is quasi-compact, generically closed, and contains $\min R$. We claim that $C \supseteq G_{f}$. If $p \notin C$, cover $C$ by open sets

$$
D\left(r_{\alpha}\right)=\left\{q \in \operatorname{Spec} R \mid r_{\alpha} \notin q\right\}
$$

for $r_{\alpha} \in p$.

Since $C$ is assumed quasi-compact, finitely many will do, say $D\left(r_{i}\right)$ for $i=$ $1, \ldots, n,-$ Then the finitely generated ideal $\sum r_{i} R$ is not contained in any prime in $C$, hence not contained in any minimal prime, thus is dense by the remark at the begining of the proof. Therefore, $p \notin G_{f}$ which establishes our claim.

$G$ is quasi-compact since it is the continuous image of the compact space Spec $Q(R)$ under the spec map [1, Exercise $1.17 \mathrm{v}, 1.21 \mathrm{i}]$. It is clear that $\Lambda(G)$ is also quasicompact and we have shown that $\Lambda(G) \supseteq \min R$, so that we may choose $C=\wedge(G)$ in the preceding paragraph. Thus $\Lambda(G) \supseteq G_{f}$, the required reverse inclusion. Consideration of the above defined $C$ establishes the remainder of the Theorem.

We may easily obtain the following well-known result: 
CoRollaRY. If $R$ is reduced, then min $R$ is compact $\Leftrightarrow G=\min R \Leftrightarrow Q(R)$ is $R$-flat $\Leftrightarrow$ each non-minimal prime contains a finitely generated dense ideal.

Proof: The paranthetical remark at the begining of the Theorem shows that $\min R$ is Hausdorff, establishing the first equivalence. For the second equivalence, if $G=\min R$ then, to check flatness locally on $G\left[6\right.$, Item 3.J], we use that $R_{p}$ is a field for each $p \in \min R$. Conversely, if $Q(R)$ is flat, then $R \subseteq Q(R)$ satisfies going down [6, Item 5.D] and we know $\min R \subseteq \bigwedge(G)$, hence $G=\min R$.

\section{CONJECTURES}

1. $G$ is generically closed.

2. $G_{f}$ is an affine subset of $\operatorname{Spec} R$ in the sense of Lazard [5, p. 112].

From torsion theory, this would imply the conjecture

3. $M(R)=M_{f}(R)$, where $M(R)$ is the maximal flat epimorphic extension of $R$ (see [5] or [7, Chapter XI, Section 4]), and $M_{f}(R)$ is the ring of quotients with respect to the filter consisting of those ideals that contain a finitely generated dense ideal.

\section{REFERENCES}

[1] M.F. Atiyah and I.G. Macdonald, Introduction to Commutative Algebra (Addison-Wesley Publ. Co., 1969).

[2] M. Hochster, 'Prime ideal structure in commutative rings', Trans. Amer. Math. Soc. 142 (1969), 43-60.

[3] James A. Hukaba, Commutative Rings with Zero Divisors (Marcel-Dekker). (to appear) .

[4] Joachim Lambek, Lectures on Rings and Modules (Blaisdell Publ. Co, 1969).

[5] Daniel Lazard, 'Autour de la platitude', Bull. Soc. Math. France 97 (1969), 81-128.

[6] Hideyuki Matsumura, Commutative Algebra, 2nd Ed. (Benjamin/Cummings Publ. Co., 1980).

[7] Bo Stenström, Rings of Quotients, Grundlehren Math. Wiss. Band 217 (Springer-Verlag, Berlin, Heidelberg, New York, 1975).

1009 Woodlawn Ave., Springfield, OH 45504 Unites States of America.
Department of Mathematics and Statistics Queen's University Kingston, Ontario, K7L 3N6

Canada 